\title{
APOTEMNOPHILIA SYNDROME DUE TO CHRONIC CANNABIS USE
}

\section{Serhat TUNC ${ }^{1}$, Ece BUYUKSANDALYACI ${ }^{2}$, Hamit Serdar BASBUG ${ }^{3}$}

${ }^{1}$ Department of Psychiatry, Kafkas University, Kars, TURKEY

${ }^{2}$ Bakirkoy Training and Research Hospital for Psychiatry, Neurology and Neurosurgery, Department of Psychiatry, Istanbul, Turkey.

${ }^{3}$ Department of Cardiovascular Surgery, Kafkas University, Kars, TURKEY

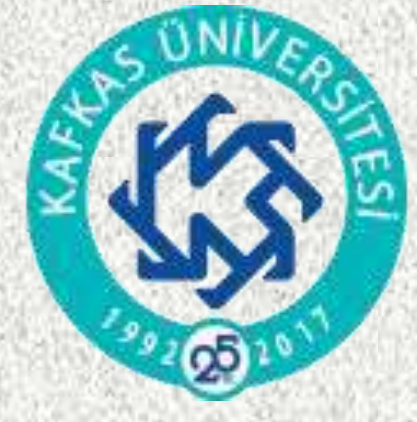

Apotemnophilia is an unusual condition which is defined as the strong and persistent desire to lose healthy extremities by amputation or seek to be paraplegic. It was first introduced by John Money in 1977. The etiology of Apotemnophilia is not yet wholly specified despite suggested several etiological mechanisms including psychiatric and neurological components. In this paper, a patient with apotemnophilia findings which is induced by using cannabis intensively for four years was reported.

A 22-year-old male patient was referred with an extreme demand of amputation of his left hand from the admission of cardiovascular surgery (CVS) department where he was immediately sent to the psychiatry outpatient clinic. Although cannabis use was reported, no orthopedic or neurological abnormality was detected in the physical examination.

Cannabis use was stopped. Alprazolam $2 \mathrm{mg}$ /day and selective serotonin reuptake inhibitor (SSRI) (escitalopram, $5 \mathrm{mg}$ /day) was initially prescribed, and the dose was increased gradually (up to $20 \mathrm{mg} /$ day) in response to treatment. After ten days, the overall symptomatic status was significantly improved. He almost never had an urge to amputation.

This case of apotemnophilia is thought to be the cause of chronic and intensive cannabis use. Assuming that cannabis use has an impact on the chemistry or morphology of the temporal lobe, we aim to emphasize the need to support these findings with more comprehensive studies. Furthermore, investigating such curious conditions that stay in the hinterland between neurology and psychiatry may supply critical points into the question of how neural activity leads to mental problems.

Keywords: cannabis-related disorder, body image, amputation 